\title{
Button Battery Ingestion in Children: A Specific Clinical Issue
}

We read with interest the paper by Lee ${ }^{1}$ about the management of foreign-body ingestion in children. We are a referral center for foreign-body management in our area, and we would be pleased to discuss our experience. Our pediatric endoscopy unit ensures 24-hour availability. Since the year 2000 , we have managed the removal of 64 foreign bodies $(0.9 \%$ of all endoscopic examinations), among which 7 (10\%) were button batteries (BBs). The children who ingested batteries ( 4 boys and 3 girls) had a mean age of 43 months (range, 12-102 months).

All the batteries were found in the esophagus and were successfully removed endoscopically, under general anesthesia and tracheal intubation. Among the ingested batteries, 4 had been impacted for less than 24 hours and were easily removed with a 9-mm flexible endoscope, with an operative channel of $2.8 \mathrm{~mm}$ (Pentax Medical, Tokyo, Japan). The esophageal mucosa presented only a mild hyperemia after the removal. The postoperative course was devoid of complications, and the patients were discharged after administering proton pump inhibitor therapy 48 hours after endoscopy.

In the other 3 cases, the battery had been in the esophagus for more than 48 hours; thus, management was more troublesome. Two of these had already eroded the mucosa at the upper esophageal sphincter and required rigid endoscopy in cooperation with a pediatric ear, nose, and throat (ENT) physician. One patient ( 8.5 years old) presented with an esophageal perforation after the procedure and was conservatively treated with total parenteral nutrition, fasting, and antibiotics without long-term complications. The second patient (1 year old) presented a perforation that was treated similar to that in the first patient; however, he developed a severe esophageal stenosis that required 11 endoscopic pneumatic dilations and topical treatment with steroids and mitomycin C. This patient also presented elevated blood chromium levels that slowly decreased over the following months. The third patient ( 2 years old) was referred from another center where an endoscopy had been performed without removing the battery, as it was partially included within the esophageal wall. Therefore, upon arrival at our center, a computed tomography scan was performed and confirmed the location of the battery in close proximity to the pericardium. The battery was removed with a flexible endoscope in the presence of a multidisciplinary team that included pediatric surgeon, cardiothoracic surgeon, ENT physician, and pediatric anesthesiologist. No intraoperative or early complication occurred during the 15-day hospitalization.

Currently, according to the international series, ${ }^{2}$ as well as our experience, foreign-body ingestion is not frequent in childhood in developed countries. It is usually accidental and involves common objects such as BBs, which represent a peculiar risk and a challenging management. Timing of ingestion represents the major risk factor for extraction, considering that batteries retained for more than 24 hours frequently get embedded in the mucosa. As a consequence, removal with a flexible endoscope may not be feasible and the use of a rigid endoscope with specific forceps may be needed, in cooperation with a pediatric ENT physician.

Complications of BBs retained for more than 24 hours may be dangerous, mostly owing to the intrinsic properties of BBs and their effects on the esophageal wall. This is especially true for lithium batteries, the use of which has increased in recent years. Their bigger dimensions and higher charge carry a greater potential for harm. ${ }^{1}$ Life-threatening complications include esophageal perforation, and aortoesophageal and tracheoesophageal fistulas. Other severe complications include

Received: August 10, 2018 Accepted: September 5, 2018

Correspondence: Francesco Macchini

Department of Pediatric Surgery, Fondazione IRCCS Ca' Granda - Ospedale Maggiore Policlinico, Via Francesco Sforza 28, Milano 20122, Italy

Tel: +39-02-55032544, Fax: +39-02-55032546, E-mail: francesco.macchini@policlinico.mi.it

ORCID: https://orcid.org/0000-0001-7380-7700

(c) This is an Open Access article distributed under the terms of the Creative Commons Attribution Non-Commercial License (http://creativecommons.org/licenses/by$\mathrm{nc} / 3.0$ ) which permits unrestricted non-commercial use, distribution, and reproduction in any medium, provided the original work is properly cited. 
esophageal strictures, vocal cord paralysis from recurrent laryngeal nerve damage, tracheal stenosis or tracheomalacia, pulmonary infections, and spondylodiscitis. ${ }^{3}$ Finally, pneumopericardium due to esophagopericardial fistula, even if rare, has been described after an ingested BB was impacted in the distal esophagus. ${ }^{4}$ For these reasons, a multidisciplinary approach is advised, involving pediatric endoscopists, pediatric surgeons, cardiothoracic surgeons, ENT physicians, pediatric anesthesiologists, and intensivists. Furthermore, severe complications such as injury to adjacent structures and heavy metal poisoning may develop even several days after extraction, especially when BBs are retained for long periods. Hence, prolonged surveillance and follow-up are mandatory. ${ }^{5}$

In conclusion, we think that $\mathrm{BB}$ ingestion represents a specific and challenging clinical issue that requires a dedicated team in tertiary centers.

\section{Conflicts of Interest}

The authors have no financial conflicts of interest.

\section{Author Contributions}

Conceptualization: Francesco Macchini

Data curation: Elettra Vestri, Martina Ichino
Formal analysis: Anna Morandi

Investigation: MI

Methodology: AM, Giorgio Fava

Supervision: GF, Ernesto Leva

Validation: EL

Writing-original draft: FM, EV

Francesco Macchini, Elettra Vestri, Martina Ichino, Anna Morandi, Giorgio Fava and Ernesto Leva

Department of Pediatric Surgery, Fondazione IRCCS Ca' Granda - Ospedale Maggiore Policlinico, Milano, Italy

\section{REFERENCES}

1. Lee JH. Foreign body ingestion in children. Clin Endosc 2018;51:129136.

2. Centers for Disease Control and Prevention (CDC). Injuries from batteries among children aged $<13$ years--United States, 1995-2010. MMWR Morb Mortal Wkly Rep 2012;61:661-666.

3. Ventura F, Candosin S, Barranco R, et al. A fatal case of coin battery ingestion in an 18-month-old child: case report and literature review. Am J Forensic Med Pathol 2017;38:43-46.

4. Soni JP, Choudhary S, Sharma P, Makwana M. Pneumopericardium due to ingestion of button battery. Ann Pediatr Cardiol 2016;9:94-95.

5. Kramer RE, Lerner DG, Lin T, et al. Management of ingested foreign bodies in children: a clinical report of the NASPGHAN Endoscopy Committee. J Pediatr Gastroenterol Nutr 2015;60:562-574. 